\title{
Mujeres sentenciadas por homicidio en la ciudad de México
}

\author{
Elena Azaola \\ Centro de Investigaciones y Estudios Superiores en Antropología Social \\ Juárez 87, Tlalpan. México 14000 D.F.
}

\section{Resumen}

El artículo aborda de manera sintética los resultados de un estudio comparativo emprendido entre hombres y mujeres sentenciados por homicidio en la Ciudad de México, enfocando particularmente la situación de las mujeres.

El estudio incluyó la revisión de 400 expedientes de hombres sentenciados por homicidio, que representan al 43 por ciento de los convictos por este delito en la ciudad de México. Nuestra muestra comprendió también a las 50 mujeres sentenciadas por homicidio en esta ciudad. Además de haber tenido acceso a los expedientes, se realizaron entrevistas en profundidad con todas las mujeres con el fin de realizar una reconstrucción de sus historias de vida. Nos interesaba conocer los tipos de homicidio más frecuentemente cometidos por varones y contrastarlos con aquellos que encontramos entre las mujeres. También nos interesaba conocer si existían diferencias en el modo como la justicia trata a ambos. En los dos casos encontramos información relevante que no había sido tratada por otros estudios en México, como es el hecho de que las mujeres tengan una sentencia un 25 por ciento más prolongada que los varones por el mismo delito en la ciudad de México.

Palabras clave: mujeres, sentencias, homicidio, historias de vida.

\section{Abstract. Women convicted for homicide in Mexico City}

The article provides a brief summary of the results of a comparative study between men and women serving time for homicide in Mexico City, with a particular focus on women inmates.

The study included a review of the records of 400 men convicted of homicide, with was 43 per cent of those serving time in Mexico City prisons for such crime. Our sample also comprised the 50 women prisoners in Mexico City convicted of homicide. We enjoyed full access to files regarding the prisoners and conducted in-depth interviews with all of the women in an effort to reconstruct their life stories. We undertook this review in an effort to record the types of homicide most frequently commited by men and contrast those with trends among the women prisoners. We also hoped to determine whether differences existed in the way the system of justice dealt with men and women. In both cases we uncovered relevant information that had not been dealt with in previous mexican studies, such as the fact that in homicide cases women draw a sentence that is 25 per cent longer than that of their male counterparts in Mexico City.

Key words: women, sentences, homicide, life stories. 
El presente trabajo expone de manera abreviada algunos de los resultados que obtuvimos en un estudio comparativo entre hombres y mujeres sentenciados por el delito de homicidio en la ciudad de México, y se detiene, particularmente, en la situación de las mujeres ${ }^{1}$. El estudio sobre estas últimas se llevó a cabo durante 1994 al interior del Centro de Readaptación Social Femenil, que se ubica en Tepepan, al sur de la ciudad de México. Antes de penetrar en la descripción de este universo, quisiéramos proporcionar algunos datos que pueden ser útiles para contextualizar el problema.

Para 1994 México, un país de casi 90 millones de habitantes, tenía una población total de 91.788 internos en todos los centros penitenciarios del país, de los que el 96,4\% eran hombres y sólo el 3,6\% mujeres. En el Distrito Federal, la ciudad capital con más de 10 millones de habitantes, la población era de 7.855 internos, de los que el $95,3 \%$ eran hombres y el $4,7 \%$ mujeres $^{2}$. Esta escasa participación de la mujer en el delito es un fenómeno que no solamente ocurre en nuestro país, pues, como es bien sabido, es muy raro que la proporción de mujeres exceda al $15 \%$ de la población en prisión, por lo cual este hecho ha sido a menudo citado por los especialistas como una evidencia de que las medidas de control informal resultan ser mucho más severas y efectivas para con la mujer (Smart, 1976; Zaffaroni, 1993; Larrauri, 1994).

Por lo que se refiere a la tasa de muertes por homicidio, México tiene una elevada tasa de 19,7 por 100 mil habitantes, que resulta muy superior a la de otros países, pero que, sin embargo, se ha mantenido relativamente estable de 1970 a la fecha, mientras que, por ejernplo, Estados Unidos ha visto con preocupación que su tasa se incrementara de 4,5 a 9,5 durante el periodo que va de 1960 a la fecha. En números absolutos, mientras que para 1990 se reportaron en Estados Unidos un total de 23.440 homicidios, en México durante los últimos años se han reportado alrededor de 16.000, la mitad de los cuales son de tipo intencional, mientras que la otra mitad son los accidentales o culposos ${ }^{3}$. En promedio, casi una quinta parte de la población total de las prisiones en México se encuentra interna por el delito de homicidio. Éste es también el caso en el Distrito Federal, en donde, para 1994, había un total de 931 hombres y 50 mujeres que ya habían obtenido una sentencia por el delito de homicidio, habiendo otra cantidad similar: que se encontraba en proceso por el mismo delito.

Nuestro universo de estudio lo conformaron las 50 mujeres, es decir, el $100 \%$ de las sentenciadas por homicidio en la ciudad de México, con quienes se trabajó a profundidad en entrevistas abiertas que tenían por objeto la ela-

1. AzAOLA, Elena: El delito de ser mujer. Hombres y mujeres homicidas en la Ciudad de México: bistorias de vida, en prensa.

2. En todos los casos, las cifras que se proporcionan en este estudio provienen de fuentes oficiales tales como: las Estadísticas Penitenciarias de la Secretaría de Gobernación, o las de la Dirección General de Reclusorios del Distrito Federal, México, 1994.

3. WOLFGANG; FERRACUTI, 1971: 291-292, asi como: "Murder. Especial Report", en: Newsweek, 15 de agosto de 1994: 8-16. 
boración de historias de vida, además de haber tenido acceso a la consulta de sus expedientes ${ }^{4}$. El levantamiento de las historias de vida, además de ser un instrumento útil para el mejor entendimiento de cada caso en lo individual, también hizo posible vislumbrar algunos de los rasgos que un gran número de mujeres sentenciadas tiene en común y a los que más adelante haremos referencia.

El estudio también incluyó la consulta de 400 expedientes de hombres sentenciados por homicidio, que representan el $43 \%$ del total de los sentenciados por este delito en la ciudad de México. Esta consulta tuvo por objeto elaborar una tipología de los homicidios que con mayor frecuencia comete el hombre, a fin de poderlos contrastar con aquellos que comete la mujer. Asimismo, nos interesaba saber si ambos reciben un trato similar por parte de la justicia. En ambos casos obtuvimos información relevante que no ha sido abordada por otros estudios (Ruíz Harrell, 1973; Flanet, 1985; Hernández Bringas, 1989; Rodríguez Manzanera, 1990; Islas, 1991; Osorio y Nieto, 1992). En efecto, a partir de la lectura de los expedientes, propusimos que los homicidios cometidos por la población de hombres que analizamos, podía clasificarse de la siguiente manera: el $49 \%$ como homicidios cometidos en riña; el $28 \%$ en asalto; el $8 \%$ en contra de algún familiar; el $4 \%$ por abuso de alguna autoridad y el $11 \%$ por varios motivos distintos a los anteriores.

Este panorama contrasta fuertemente con el que obtuvimos entre las mujeres y que constituye el motivo del presente trabajo. En ellas encontramos que el $76 \%$ de los homicidios tiene por víctima a un familiar y sólo el $24 \%$ se dirige a una persona fuera de este núcleo. Asimismo, otro dato sorprendente fue el del trato diferencial que reciben por parte de la justicia, ya que, en promedio, mientras que el total de los hombres sentenciados por homicidio tiene una sentencia de 18,6 años, las mujeres una de 23,3 , diferencia que incluso se conserva si se comparan únicamente los homicidios cometidos por ambos en contra de familiares, pues mientras que en estos casos los hombres reciben en promedio una sentencia de 18 años, las mujeres una de 24 . Ello implica que las mujeres sentenciadas por homicidio tienen una sentencia que, en promedio, resulta ser un $25 \%$ más elevada de la que por el mismo delito obtienen los varones. Este hecho tampoco ha sido abordado hasta ahora por otros estudios (Instituto de Investigaciones Jurídicas, 1983; Villanueva, 1989; Lima Malvido, 1991; Cuevas, 1992).

Es indudable que tanto en el tipo de homicidios que ambos cometen, como en el trato que reciben por parte de la justicia, lo que puede dar cuenta de las diferencias son los patrones de conducta, de socialización, de creencias y de

4. Es preciso agregar que, a pesar de que esta reconstrucción resultó muy dolorosa para la mayoría de las mujeres, en ningún caso esto las hizo desistir, a pesar de que se les había expresado que su participación en el estudio era enteramente voluntaria. Inclusive, muchas de ellas señalaron que hacía tiempo que no habían tenido la oportunidad de hablar o reflexionar sobre lo que había ocurrido y que, durante el juicio, no se les preguntó acerca de su historia ni se les dio la oportunidad de ser escuchadas y de exponer sus puntos de vista. 
valores que sitúan en diferente posición al hombre y a la mujer, y que una y otra vez aparecen claramente enunciados en las historias de vida que recogimos. El hecho de que nuestra sociedad no perciba de la misma manera un acto de agresión si éste proviene de un hombre que de una mujer, o el hecho de que en el primero se considere a estas conductas casi como inevitables o parte de su "naturaleza», mientras que en la segunda como algo que subvierte su «esencia», o bien el hecho de que no se asigne el mismo peso ni se otorgue el mismo reproche para quien se considera un mal padre que para una mala madre, constituyen sólo algunas de las expresiones de las diferencias de género que se observan nítida y cotidianamente en este campo.

Otros hechos también dan cuenta de estas diferencias. Por ejemplo, el hecho de que el mayor número de homicidios que cometen los hombres ocurran en espacios abiertos, mientras que los de la mujer tienen lugar adentro de la casa, da cuenta de manera palpable de una mayor y más significativa presencia del hombre, en el mundo exterior, en tanto que la mujer parece confinada al ámbito familiar.

Después de la lectura de numerosos expedientes, queda igualmente claro que, las más de las veces, los homicidios cometidos por el hombre tienen por víctima a otro hombre joven con el cual se riñe por un motivo cualquiera, puesto que, lo que en realidad se disputa, es el deseo de mostrar, de dejar una evidencia que elimine, que anule de una vez por todas, cualquier duda acerca de la propia superioridad, la propia fuerza. En la mujer, en cambio, el homicidio parece ser el desenlace de múltiples episodios de violencia en los que, algunas veces, ella ha ocupado el lugar de víctima, otras, de victimario, y en donde los motivos parecen más complejos y difíciles de descifrar, pues sólo en apariencia subvierten su ser-mujer (véase Lagarde, 1993).

Dejaremos aquí los contrastes entre la conducta violenta del hombre y de la mujer, para poder penetrar en los 50 casos de homicidio que estudiamos. Comenzaremos por los 38 casos en que las mujeres dieron muerte a familiares y que representan, como hemos dicho, el $76 \%$ del total. Este grupo de homicidios puede clasificarse de la siguiente manera:

- 26 casos de homicidio a niños, que representan el $52 \%$ del total. En el $65 \%$ de estos casos las mujeres dieron muerte a sus propios hijos y en el $35 \%$ restante a hijos de su pareja, o bien a niños que habían adoptado, les habían «regalado» o se hallaban bajo su cuidado por ser hijos de algún familiar (sobrinos, nietos, hermanos).

- 11 casos de homicidio a esposos o parejas de las mujeres sentenciadas, que representan el $22 \%$ del total, y

- 1 caso de homicidio a la madre de una mujer interna, que representa el $2 \%$ del total.

Como en cuatro casos las mujeres dieron muerte a todos sus hijos, el número total de víctimas de ese grupo fue de 32 niños, de los que el $44 \%$ eran varones y el $56 \%$ mujeres. En cuanto a la edad, el $75 \%$ tenía entre 0 y 5 años; $12,5 \%$ entre 6 y 10 , y otro $12,5 \%$ entre 11 y 16 años. El homicidio a niños 
no es, lamentablemente, un fenómeno extraño en nuestro país. Un estudio reciente, elaborado a partir de la base de datos sobre mortalidad de la Secretaría de Salud, ha puesto de manifiesto que, durante la década de los ochenta, se registraron en la República Mexicana 2.939 muertes por homicidio entre menores de 0 a 4 ańos, lo que supone que, en promedio, un niño menor de 4 años muere por homicidio cada dos días en México (Híjar-Medina y otros, 1994: 529-532). Por su parte, Ruíz Harrell seńaló que, durante 1994, se reportaron en centros hospitalarios de la ciudad de México un total de 2.372 casos de niños menores de 10 años que fueron lesionados de manera intencional, de los que sólo en 408 casos se inició una denuncia penal (Reforma, 21 de noviembre de 1994).

No sólo llama la atención la significativa presencia de los niños, sino también el escaso peso de otros familiares que contrasta nuevamente con lo que ocurre entre los hombres. En efecto, en la muestra que analizamos, encontramos que el $49 \%$ de los casos en que los varones dan muerte a familiares, tienen por víctima a la esposa o la pareja; el $21 \%$ a sus hijos o los de su pareja; otro $21 \%$ se dirige a sus padres o abuelos; el $6 \%$ a hermanos y el $3 \%$ a otros miembros de la familia.

El hecho de que la mujer dirija la violencia principalmente hacia los hijos muestra de manera paradójica el significativo lugar que nuestra cultura asigna a la relación entre la madre y los hijos. Relación sobrecargada con toda clase de afectos y expectativas y cuya disolución resulta tan impensable que en no pocos casos la mujer que da muerte a los hijos intenta el suicidio como si, al haber aniquilado a sus hijos, hubiera dado muerte a su razón-de-ser (Rascovsky, 1974; Badinter, 1981; De Beauvoir, 1981; Basaglia, 1983; Lagarde, 1993).

Dentro del grupo de madres que dieron muerte a sus hijos nos pareció posible distinguir por lo menos dos categorías. En la primera habría que incluir aquellos niños que murieron como resultado de un largo y continuo proceso de malos tratos, negligencia, abusos, y en los que sobresale el hecho de que la madre hubiera, a su vez, recibido el mismo trato, primero, por parte de sus padres y, más tarde, de su pareja. Consideramos que cabría ubicar dentro de esta categoría a 15 de los 26 casos de homicidio a niños que estudiamos.

En una segunda categoría se encontrarían 5 de los 26 casos en los que el homicidio se produjo en un solo acto, insólito e intempestivo, en el que habría una pérdida transitoria de conciencia por parte de la madre, quien no podría dar cuenta de su acto, ya que éste se habría producido como el desenlace de un estado de tensión acumulada que, las más de las veces, se habría producido por la amenaza proveniente de la pareja de despojarla de sus hijos. De los 6 casos restantes de homicidio a niños, 4 fueron cometidos por mujeres a las que se consideró inimputables por tener severos trastornos, otro fue un caso de infanticidio (es decir, que fue cometido por la madre dentro de las 72 horas posteriores al nacimiento) y, otro más, fue el resultado de un pacto suicida por envenenamiento que la madre suscribió con cuatro hijas de 9 a 16 años y al que sobrevivieron una hija y la madre. 
En cuanto a los 11 casos de homicidio cometidos en contra de la pareja, el motivo que se alega es el maltrato, la infidelidad y, sobre todo, las continuas humillaciones que muchas veces las mujeres toleraron durante años. Había, sin embargo, dos casos de mujeres indígenas en los que todo parecía indicar que habían aceptado, o les habían hecho "aceptar», su culpabilidad para encubrir a otros miembros de la familia, quienes habrían dado muerte a su esposo para despojarlo de sus tierras. De ser esto cierto, sería todavía más baja la proporción de casos en los que la mujer dirige la violencia hacia la pareja, no obstante que la mayoría de las sentenciadas por este delito reportaron altos índices de maltrato por parte de sus esposos. Otro dato significativo es que casi una tercera parte de los esposos a los que dieron muerte eran policías.

También llama la atención el hecho de que el único homicidio cometido en contra de un ascendiente hubiera sido el acto de una mujer a quien se declaró inimputable por haber padecido desde pequena un severo trastorno psiquiátrico, lo que, por lo que a esta muestra respecta, pone de manifiesto que los padres serían, a diferencia de los hijos, una especie de valor intocable para la mujer. De nueva cuenta, esta actitud contrasta con la de los hombres de la muestra que analizamos, pues en ellos encontramos que atentan en contra de los hijos en igual proporción ( $21 \%$ de los casos, respectivamente) que en contra de los padres.

Por lo que se refiere al $24 \%$ de los homicidios cometidos por las mujeres en contra de personas ajenas a la familia, éstos pueden clasificarse de la siguiente manera:

- 4 casos de homicidio cometido en riña y bajo los efectos de alcohol, que representan el $8 \%$ del total. Estos casos son casi siempre de mujeres que ejercían la prostitución, habían sido abandondonadas y vivían prácticamente en la calle.

- 4 casos de homicidio cometido en asalto en los que la mujer casi siempre desempeñaba el papel de cómplice dentro de una banda integrada por varones y que representan el $8 \%$ del total.

- 2 casos de homicidio cometidos en contra de vecinos que representan el $4 \%$ del total.

- 1 caso de homicidio en contra de otra mujer a la que la sentenciada consideraba su rival amorosa, que representa el $2 \%$ del total.

- 1 caso de homicidio por motivos políticos que representa el $2 \%$ del total.

Lo que de este último panorama cabe destacar es la semejanza que en cuanto a las víctimas y los motivos existen en los homicidios que cometen el hombre y la mujer cuando ello ocurre fuera del ámbito familiar. Ello quiere decir que encontramos los contrastes más significativos de género en las conductas homicidas que tienen por víctimas a rniembros de la familia.

Quisiéramos ahora referirnos al perfil de las 50 mujeres sentenciadas, independientemente del tipo de homicidio que hubieran cometido 5 .

5. Obtuvimos la siguiente información, tanto de los expedientes como de las entrevistas efectuadas a las internas. 
- En cuanto a su lugar de origen, el $56 \%$ de las mujeres nacieron en el Distrito Federal, el $42 \%$ en diversos estados circundantes a la capital y el $2 \%$ en el extranjero (Guatemala).

- La edad a la que, en promedio, cometieron el delito de homicidio, es de 26 años. Sin embargo, encontramos que, en promedio, estas mujeres habían abandonado o les habían hecho abandonar su hogar a los 16 años, edad en la que la mayoría estableció su primera relación de pareja y en la que muchas tuvieron su primer embarazo.

- El 70\% de estas mujeres habían padecido formas y grados distintos de violencia, negligencia, abuso sexual o abandono por parte de su familia, mientras que el $66 \%$ lo recibieron también de sus parejas y el $60 \%$ fueron nuevamente maltratadas por la policía. Un $34 \%$ de las mujeres cometieron el homicidio momentos después de haber sufrido continuos y severos episodios de violencia por parte de sus parejas.

- Mientras que en el hombre más de un $40 \%$ de los homicidios están relacionados con el alcohol, encontramos que un $16 \%$ de las mujeres sentenciadas por homicidio tiene alguna adicción, aunque sólo el $12 \%$ cometió el delito estando bajo el efecto de alcohol o de alguna otra droga (solventes o mariguana).

- Por lo que toca a su situación socioeconómica, el 78\% de las mujeres sentenciadas por homicidio pertenece a un estrato social bajo, el $16 \%$ a uno medio y el $6 \%$ a uno alto. Dentro del primer grupo cabría distinguir a un $26 \%$ de las mujeres sentenciadas que habrían sobrevivido en condiciones de extrema pobreza.

- Del total de las mujeres sentenciadas por homicidio, el 12\% habrían sido clasificadas por las autoridades correspondientes como inimputables, mientras que al restante $88 \%$ se le consideró capaz de responder por sus actos.

- La mayoría de estas mujeres vivían en unión libre, en relaciones con frecuencia violentas e inestables, o bien habían sido abandonadas por su pareja y tenían hijos que provenían de diferentes uniones. De hecho, el $80 \%$ de las mujeres sentenciadas por homicidio son madres y tienen, en promedio, 3 hijos. Asimismo, el $42 \%$ de las mujeres que dieron muerte a sus hijos han buscado un nuevo embarazo.

- El $16 \%$ de estas mujeres intentaron, antes o después del homicidio, el suicidio.

- En cuanto a su escolaridad, el $22 \%$ de las mujeres eran analfabetas y el $26 \%$ había cursado algún grado de la primaria, mientras que $22 \%$ habían completado este ciclo escolar. El $10 \%$ había cursado la secundaria y el $20 \%$ había iniciado la preparatoria o alguna carrera técnica, mientras que sólo el $2 \%$ había llegado al primer año en una universidad.

- Antes de ingresar, las mujeres desempeñaban las siguientes ocupaciones: $24 \%$ habían laborado como empleadas domésticas; $16 \%$ se dedicaban al comercio; $14 \%$ estaban en el hogar; $12 \%$ eran secretarias o recepcionistas; $8 \%$ realizaban labores agrícolas; otro $8 \%$ ejercían la prostitución; $6 \%$ ha- 
bían sido empleadas de banco; $4 \%$ empleadas de gobierno; $2 \%$ maestras; $2 \%$ costureras; $2 \%$ obreras, y otro $2 \%$ albañiles.

- Las ocupaciones que, por su parte, ejercía la pareja de estas mujeres, son: $23 \%$ obreros; $17 \%$ policías; $17 \%$ empleados de banco o del gobierno; $15 \%$ comerciantes; $13 \%$ albañiles; $12 \%$ mecánicos; $4 \%$ braceros; $4 \%$ campesinos y $2 \%$, respectivamente, estudiantes, choferes y desempleados.

- En promedio, las mujeres sentenciadas por homicidio cumplen una sentencia de 23,3 años. A diferencia de lo que ocurre con los varones, no existen casos de mujeres que hubieran reincidido en el delito de homicidio y sólo un $4 \%$ de estas mujeres tenía ingresos previos por otros delitos (robo, contra la salud o lesiones).

- El número total de víctimas fue de 57, de las que: 32 (56\%) fueron los hijos $\mathbf{u}$ otros niños que se hallaban bajo el cuidado de las mujeres sentenciadas; 11 (19\%) eran sus esposos o compañeros; 4 (7\%) eran desconocidos a los que dieron muerte en riña; $3(5 \%)$ eran compañeros de trabajo; 2 eran mujeres a las que consideraron sus rivales ya que se disputaban a la pareja; 2 eran sus vecinos; 2 eran las patronas a las que dieron muerte en asalto y 1 era su madre.

- Estas 57 víctimas fueron muertas de la siguiente manera: $35 \%$ por golpes o «caídas»; $26 \%$ por arma blanca; $21 \%$ por arma de fuego; $9 \%$ por envenenamiento y otro $9 \%$ por ahorcamiento.

Mencionaremos a continuación algunas de las conclusiones que nos fue posible obtener del estudio que aquí hemos expuesto de manera necesariamente breve y esquemática ${ }^{6}$.

1. El estudio acerca de las conductas homicidas, que fue elaborado desde una perspectiva de género, se ha mostrado capaz de revelar aspectos que han sido poco abordados por otros estudios en nuestro país ${ }^{7}$. Entre ellos, la existencia de diferencias significativas en las sentencias que por el mismo tipo de homicidios se otorgan al hombre y a la mujer, en detrimento de esta última. Asimismo, los rasgos que una y otra vez se repiten en las historias de las mujeres, nos confirman en la pertinencia de abordar y profundizar el estudio del homicidio como un hecho social, como un fenómeno cultural.

2. Uno de los rasgos que encontramos como distintivo de la conducta homicida en el hombre, es que ésta parece ser una manifestación extrema de la rivalidad, la competencia frente a los pares. Asimismo, parece vincularse más a estallidos violentos momentáneos que a acciones calculadas de tiem-

6. Remitimos al lector a la consulta del estudio arriba citado, en donde encontrará los elementos en los que pretendemos fundar las conclusiones siguientes.

7. Como se sabe, a partir de los trabajos de Stoller (1968) y Millet (1975), el enfoque de género se ocupa por abordar la diferencia sexual en tanto que diferencia social. En otros términos, se ha dicho que el género es la construcción imaginaria que, a partir de las diferencias sexuales, elabora la sociedad. 
po atrás. Estos y otros elementos, sumados al hecho de que el hombre comete el homicidio principalmente en espacios abiertos y contra personas que no son miembros de su familia, nos hablan de una mayor presencia objetiva y subjetiva del hombre en el mundo, de su pertenencia a un universo de relaciones que rebasa el ámbito familiar, de su inscripción en el mundo de la rivalidad, la competencia, del deseo por alcanzar, preservar o imponer un lugar con base en la fuerza o la superioridad de las armas. Todo ello nos habla del lugar desde el cual, la posición desde la cual, el hombre, a diferencia de la mujer, es socializado e introducido a nuestra cultura desde pequeño, y aun antes de nacer; en otros términos, a las diferencias de género. Lugar desde donde continuamente se le enseña al varón a dirimir los conflictos de manera violenta, a imponer su voluntad por encima de la de los demás, a no tolerar las diferencias, a abusar de la debilidad, a valorar y hacer deseable la posición desde la cual el poderoso abusa de su poder, en fin, a aniquilar al otro de manera simbólica, pero a veces también literal.

3. En contraste, lo que en la conducta homicida distingue a la mujer, es el peso significativo que tienen sus familiares, particularmente los hijos. Este peso revela, aun cuando parezca un contrasentido, el lugar de privilegio que nuestra cultura otorga a la maternidad. Lugar de privilegio que, al ser puesto en cuestión, al poner al descubierto la ambivalencia que suscita la maternidad, recibe las sanciones más severas, las más elevadas sentencias. Como si con ello quisiéramos asegurarnos que el fenómeno permanecerá confinado, que nada tiene que ver con nosotros, que no nos pertenece y que ni siquiera habría lugar para que nos interrogáramos. Castigo ejemplar que, a la par que pone de manifiesto el valor que nuestra cultura concede a la maternidad, soslaya el cúmulo de violencias previamente recibidas por la mujer. Se trataría, entonces, de muertes que a menudo son la culminación de un largo proceso de tortura, proceso en el que la mujer unas veces ha sido el sujeto activo, otras, el pasivo. Muertes que la mujer dirige hacia aquellos a quienes ha amado en exceso, que tienen para ella una importancia capital, de quienes depende en forma radical o bien, que dirige a aquellos para quienes existe un odio silenciado, diferido. Muertes también que, tras aniquilar la-razón-de-ser de la mujer, la aniquilan como ente social, que le imponen un desgarramiento brutal, desvastador, que suponen el destierro de su condición social, de su condición de mujer, que, en suma, ponen en evidencia que la mujer, al tiempo que mata, muere. De ser esto así, el homicidio en el hombre sería un acto que, en la medida en que responde a un odio fugaz, pasajero, sería, desde esta perspectiva, un acto compatible con su identidad, un acto por el que incluso en ciertos ámbitos puede esperar cierto tipo de reconocimiento, un acto, en suma, compatible con su identidad, con su «ser hombre». En sentido opuesto, para la mujer se trataría de actos que radicalmente la aniquilan, que subvierten su identidad, su «ser mujer»; que marcarían, entonces, el "destierro" de su condición de mujer. 


\section{Bibliografía}

AZAOLA, Elena (1994). El delito de ser mujer. Hombres y mujeres homicidas en la Ciudad de México: historias de vida. En prensa.

BADINTER, Elizabeth (1981). ¿Existe el amor maternal? Barcelona: Paidós.

BASAGLIA, Franca (1983). Mujer, locura y sociedad. México: Universidad Autónoma de Puebla.

BEALVOIR, Simone de (1981). La mujer rota. Buenos Aires: Hermes.

Cuevas SOSA, Alejandro (1992). La mujer delincuente bajo la ley del hombre. México: Pax.

FLANET, Véronique (1985). La madre muerte. Violencia en México. México: Fondo de Cultura Económica.

HERNÁNDEZ BRINGAS, Héctor (1989). Las muertes violentas en México. México: UNAMCRIM.

Hijar-Medina, Martha C., y otros (1994). "Mortalidad por homicidio en niños. México, 1979-1990n. Salud Pública, 36: 529-537, México.

IsLAS, Olga (1991). Análisis lógico de los delitos contra la vida. México: Trillas.

INSTITUTO DE INVESTIGACIONES JURIDICAS (1983). La mujer delincuente. México: UNAM.

JosÉ, Cristina (1985). Women doing life sentences. A phenomenological study. Tesis. Universidad de Michigan.

LAGARDE, Marcela (1993). Los cautiverios de las mujeres: madresposas, monjas, putas, presas y locas. México: UNAM.

LARRAURI, Elena (comp.) (1994). Mujeres, derecho penal y criminologia. Madrid: Siglo XXI Editores.

LIMA MALVIDO, Ma. de la Luz (1991). Criminalidad femenina: teorias y reacción social. México: Porrúa.

Osorio y Nieto, César Augusto (1992). El homicidio. México: Porrúa.

RascovsKY, Arnaldo (1974). El filicidio. Buenos Aires: Orión.

Rodriguez Manzanera, Luis (1990). "El homicidio en México». En AnIYAR DE CASTRO (ed.). Criminología en América Latina. ONU, Roma, mayo: 149-157.

RUIZ HARRELL, Rafael (1973). El homicidio y la violencia como factores de salud pública en México. México: s/e.

SMART, Carol (1976). Women, crime and criminology. Londres: Routledge and Kegan Paul.

VILLANUEVA, Ruth (1989). “La mujer homicida en el Distrito Federal». En: VILLANUEVA y LABASTIDA. Dos reflexiones jurídico criminológicas. México: Librería Parroquial de Clavería.

WolfGang, M.; Ferracuti, F. (1971). La subcultura de la violencia. México: Fondo de Cultura Económica.

ZaFfaroni, Raúl (1993). "La mujer y el poder punitivo». En: Facio y otros. Sobre patriarcas, jerarcas, patrones y otros varones. Costa Rica: Ilanud. 